\title{
Injuria miocárdica postcirugía no cardiaca (MINS) en pacientes sometidos a cirugía vascular: artículo de revisión
}

\author{
Myocardial injury after noncardiac surgery (MINS) in major \\ vascular surgery, a review
}

Roberto Coloma', Katia González

\begin{abstract}
Vascular surgery is considered high risk, due to the characteristics of patients and surgical procedures. Recently, the diagnosis of myocardial injury after noncardiac surgery (MINS) was introduced to focus attention on the prognostic relevance of elevated ischemic troponin after non-cardiac surgery. In the study Vascular Events In Noncardiac Surgery Patients Cohort Evaluation (VISION), that included more than 15,000 patients who underwent non-cardiac surgery, MINS was independently associated with mortality 3 to 4 times greater than 30 days after surgery. Biccard et al published a study derived from the study VISION in which 502 patients underwent vascular surgery, resulting in significantly higher mortality $(12.5 \%$ vs $1.5 \%, p<0.001)$ in patients who developed MINS compared to those who did not develop MINS (OR 9.48, 95\% CI, 3.46-25.96). The 2014 guidelines for preoperative cardiovascular evaluation of the American College of Cardiology/American Heart Association (ACC/AHA) do not recommend the postoperative surveillance of troponin in patients without symptoms or signs of myocardial ischemia, nor in patients at high risk of coronary disease (as patients undergoing vascular surgery). On the other hand, the 2017 guidelines of the Canadian Cardiovascular Society on Perioperative Cardiac Risk, evaluation and treatment for non-cardiac surgery patients, recommend obtaining daily measurements of troponin for 48 to 72 hours after of non-cardiac surgery, in patients with a baseline risk of more than $5 \%$ of cardiovascular death or acute non-fatal myocardial infarction at 30 days after surgery. MINS is a potentially useful marker of adverse postoperative outcomes and its detection could provide opportunities to improve clinical outcomes in affected patients.
\end{abstract}

Key words: Vascular

surgery,

MINS,

myocardial injury

División de Anestesiología. Escuela de Medicina, Pontificia Universidad Católica de Chile. Santiago, Chile.

Fecha de recepción: 15 de mayo de 2019

Fecha de aceptación: 22 de junio de 2019

ORCID

https://orcid.org/0000-0003-0642-8078

Correspondencia:

Roberto Coloma

Email: ricoloma@uc.cl 


\section{RESUMEN}

La cirugía vascular es considerada de alto riesgo, debido a características propias de los pacientes y de los procedimientos quirúrgicos. Recientemente, se introdujo el diagnóstico de injuria miocárdica postcirugía no cardiaca (MINS) para centrar la atención en la relevancia pronóstica de la elevación de la troponina isquémica después de una cirugía no cardíaca[6]. Dos grandes estudios[6],[7] han demostrado que la mortalidad es significativamente mayor en pacientes que desarrollaron MINS. Las guías 2014 de evaluación cardiovascular preoperatoria de American College of Cardiology/American Heart Association (ACC/AHA)[1], no recomiendan la vigilancia postoperatoria de troponina en pacientes sin síntomas ni signos de isquemia miocárdica ni tampoco en pacientes con alto riesgo de infarto al miocardio (como pacientes sometidos a cirugía vascular)[1]. Sin embargo, las guías de sociedad cardiovascular canadiense (publicadas en 2017) sobre riesgo cardíaco perioperatorio, evaluación y tratamiento para pacientes de cirugía no cardíaca[10] recomiendan obtener mediciones diarias de troponina durante 48 a 72 horas después de cirugía no cardíaca, en pacientes con un riesgo basal superior al $5 \%$ de muerte cardiovascular o infarto agudo al miocardio no fatal a 30 días después de una cirugía. MINS se debe considerar como un marcador potencialmente útil de resultados postoperatorios adversos y su detección podría brindar oportunidades para mejorar los resultados clínicos en los pacientes afectados.
Palabras clave: Cirugía

vascular,

MINS,

injuria miocárdica.
Introducción

a cirugía vascular es considerada de alto riesgo (excepción de la endarterectomía carotídea)[1] debido a características propias de los pacientes y de los procedimientos quirúrgicos.

Los factores de riesgo de los pacientes sometidos a cirugía vascular son múltiples: edad avanzada, tabaquismo y enfermedad ateroesclerótica y entre los factores asociados a los procedimientos quirúrgicos destacan la inestabilidad hemodinámica asociada a pérdida sanguínea, clampeo aórtico, fenómenos de reperfusión y complicaciones tromboembólicas.

Las complicaciones cardiovasculares perioperatorias de este grupo de pacientes, infarto agudo al miocardio intrahospitalario, insuficiencia cardiaca congestiva y arritmias tienen una incidencia que oscila entre un 6 y que alcanza un 18\% luego de una reparación de aneurisma aórtico abierto[2]. Esta injuria miocárdica presenta una alta mortalidad durante los 30 días postoperatorio.

\section{Injuria miocárdica postcirugía no cardiaca (MINS)}

Estudios prospectivos recientes han demostrado que la elevación postoperatoria de troponina T plas- mática se asocia con un mayor riesgo de mortalidad a los 30 días después de las cirugías no cardíacas[3]-[5]. Recientemente, se introdujo el diagnóstico de injuria miocárdica postcirugía no cardiaca (MINS) para centrar la atención en la relevancia pronóstica de la elevación de la troponina isquémica después de cirugía no cardíaca[6]. Los criterios diagnósticos de MINS comprenden un nivel plasmático máximo de troponina $T$ (TnT) de cuarta generación de 0,03 ng/ml o superior que se considera debido a isquemia miocárdica, producido dentro de los 30 días después de cirugía no cardiaca[6]. Botto F y cols., publicaron el estudio Vascular Events In Noncardiac Surgery Patients Cohort Evaluation (VISION)[6] que incluyó a más de 15.000 pacientes que se sometieron a cirugía no cardíaca, MINS se asoció de forma independiente a una mortalidad 3 a 4 veces mayor a 30 días de la cirugía[6].

\section{MINS en cirugía vascular}

Recientemente Biccard B y cols.[7], publicaron un estudio derivado del estudio VISION[6] en el cual incluyeron 502 pacientes sometidos a cirugía vascular, de una cohorte de 15.102 sometidos a cirugía no cardiaca. Este estudio incluyó pacientes mayores de 45 años, sometidos a cirugías electivas o de urgen- 
cia/emergencia, que requirieron anestesia general o regional y al menos 1 noche de hospitalización después de la cirugía. A todos los pacientes les midieron concentración plasmática de TnT de cuarta generación (ensayo Elecsys TnT de Roche) a las 6 a 12 horas después de la operación y durante los primeros 3 días después de la cirugía (mediciones a las 24, 48 y 72 horas). Se excluyeron pacientes cuya etiología de elevación de la enzima pudiese haber sido de origen no cardiaco (sepsis, TEP, miocarditis). A los pacientes con una concentración de TnT en plasma de 0,04 ng/ml o mayor, se los evaluó para detectar síntomas y/o signos de isquemia miocárdica y hallazgos de isquemia en el electrocardiograma (ECG). Si un paciente con un TnT de 0,04 ng/ml o más no tenía síntomas o signos isquémicos ni hallazgos de ECG isquémicos, se les solicitó una imagen cardiaca. Las características isquémicas se definieron como cualquiera de los siguientes: un síntoma o signo clínico de isquemia miocárdica, un hallazgo de isquemia en el ECG, una alteración nueva de la motilidad de alguna pared cardíaca en la ecocardiografía o un nuevo defecto en una imagen de medicina nuclear (Tabla 1).

Los procedimientos quirúrgicos vasculares a los cuales de sometieron los pacientes fueron: reconstrucción de la aorta torácica, reconstrucción aortoilíaca, reconstrucción arterial periférica sin clampeo aórtico, cirugía cerebrovascular extracraneal o reparación aórtica endovascular.
Al comparar las características clínicas basales de los pacientes sometidos a cirugía vascular se observó: los pacientes con MINS eran significativamente mayores y con mayor frecuencia tenían fibrilación auricular, insuficiencia cardíaca congestiva, enfermedad coronaria y disfunción renal, y tenían una frecuencia cardíaca preoperatoria media más alta que los pacientes que no desarrollaron MINS. La cirugía no electiva y la revascularización de extremidades inferiores fueron más comunes en pacientes que desarrollaron MINS, mientras que la cirugía cerebral extracraneal y la reparación aórtica endovascular fueron más comunes en pacientes que no desarrollaron MINS.

De los 500 pacientes sometidos a cirugía vascular fallecieron 8 (3,6\%; IC 95\%, 2,3-5,6), dentro de los 30 días posteriores a la cirugía, en comparación con 250 de 14.584 pacientes sometidos a otras cirugías no cardiacas en la cohorte VISION (1,7\%; IC 95\%, $1,5-1,9)(p=0,005)$. La Tabla 2 muestra la mortalidad a 30 días para los pacientes quirúrgicos vasculares con y sin MINS. La mortalidad fue significativamente mayor $(12,5 \%$ vs $1,5 \%$; $p<0,001)$ en pacientes que desarrollaron MINS en comparación con aquellos que no desarrollaron MINS (OR 9,48; IC del 95\%, 3,4625,96). El análisis de regresión multivariable sugirió que el impacto de MINS en la mortalidad a los 30 días fue similar después de la cirugía vascular y no vascular ( $p=0,573$ para prueba de interacción).

Los pacientes con MINS también tuvieron tasas

Tabla 1. Características isquémicas después de cirugía vascular en pacientes con una concentración máxima de troponina T plasmática de $0,04 \mathrm{ng} / \mathrm{ml}$

\begin{tabular}{lc}
\hline Característica isquémica & Frecuencia n (\%) \\
Dolor torácico & $9(11,1 \%)$ \\
Dolor en el cuello/mandíbula/brazo & $2(2,5 \%)$ \\
Disnea & $12(14,8 \%)$ \\
Edema pulmonar & $9(11,1 \%)$ \\
Cualquiera de los de arriba & $21(25,9 \%)$ \\
Ondas Q nuevas & $1(1,2 \%)$ \\
Elevación del ST & $1(1,2 \%)$ \\
Infradesnivel ST & $16(19,8 \%)$ \\
Inversión de la onda T & $25(30,9 \%)$ \\
Bloqueo completo de rama izquierda nuevo & 0 \\
Anormalidad nueva en la motilidad de una pared miocárdica en el ECG & $2(2,5 \%)$ \\
Presunta anormalidad nueva en la motilidad de una pared miocárdica en el ECG & $8(9,9 \%)$ \\
Nuevo defecto de flujo en imagen de medicina nuclear & $1(1,2 \%)$ \\
Presunto nuevo defecto de flujo en imagen de medicina nuclear & $2(2,5 \%)$ \\
\hline
\end{tabular}


Tabla 2. Mortalidad a 30 días en pacientes sometidos a cirugía vascular

\begin{tabular}{|c|c|c|}
\hline & \multicolumn{2}{|c|}{ Mortalidad a 30 días } \\
\hline & $\mathrm{n} / \mathrm{N}$ & $\%(95 \% \mid C)$ \\
\hline Todos los pacientes de cirugía vascular & $18 / 500$ & $3,6(2,3-5,6)$ \\
\hline NO MINS & $6 / 404$ & $1,5(0,7-3,2)$ \\
\hline MINS & $12 / 96$ & $12,5(7,3-20,6)$ \\
\hline \multicolumn{3}{|c|}{ Pacientes con MINS con una concentración máxima de troponina T plasmática $\geq 0,04 \mathrm{ng} / \mathrm{ml}$} \\
\hline MINS sin características isquémicas & $5 / 41$ & $12,2(5,3-25,5)$ \\
\hline MINS con 1 o más características isquémicas & $6 / 40$ & $15(7,1-29,1)$ \\
\hline
\end{tabular}

\begin{tabular}{|c|c|c|c|}
\hline \multirow[b]{2}{*}{ Outcome } & \multirow{2}{*}{$\begin{array}{c}\text { No MINS, } \mathbf{n}=404 \\
n(\%)\end{array}$} & \multirow{2}{*}{\multicolumn{2}{|c|}{$\begin{array}{c}\text { MINS, } \mathbf{n}=\mathbf{9 4} \\
\text { OR no ajustado ( } 95 \% \text { IC), vs } \\
\text { no MINS }\end{array}$}} \\
\hline & & & \\
\hline Paro cardiaco no fatal & $1(0,2)$ & $4(4,3)$ & $17,91(1,98-162,16)$ \\
\hline Insuficiencia cardiaca congestiva & $8(2,0)$ & $11(11,7)$ & $6,56(2,56-16,81)$ \\
\hline Accidente cerebrovascular & $6(1,5)$ & $6(6,4)$ & $4,52(1,43-14,35)$ \\
\hline $\begin{array}{l}\text { Revascularización miocárdica (angioplastía o } \\
\text { bypass coronario) }\end{array}$ & $1(0,2)$ & $6(6,4)$ & $27,48(3,27-231,11)$ \\
\hline Mortalidad $(\mathrm{N}=500)$ & $6 / 404(1,5)$ & $12 / 96(12,5)$ & $9,48(3,46-25,96)$ \\
\hline
\end{tabular}

más altas de paros cardíacos no fatales, insuficiencia cardíaca congestiva, accidente cerebrovascular y revascularización miocárdica a 30 días que los pacientes sin MINS (Tabla 3).

Los pacientes quirúrgicos vasculares que sufrieron MINS tuvieron una mayor estadía hospitalaria (17 días; IQR 5-31 días) en comparación con los pacientes que no sufrieron MINS (duración de la estancia 6 días; IQR 3-10 días), $p<0,001$. Veintiuno (25,9\%) de los pacientes con MINS tuvieron síntomas clínicos isquémicos. La mortalidad a 30 días no fue significativamente diferente $(p=0,76)$ entre los pacientes con MINS con (15,0\%; IC de 95\%, 7,1-29.1; OR, 11,71; IC de $95 \%, 3,58-38,27)$ y sin una característica isquémica (12,2\%; IC del95\%, 5,3 a 25,5; OR: 9,21; IC de $95 \%, 2,68$ a 31,67). La proporción de pacientes de cirugía vascular que desarrollaron MINS que no hubiesen sido detectados sin medición rutinaria de troponina T postoperatoria (es decir, MINS asintomático) fue de 60 de 81 (74,1\%; IC de 95\%, 63,6-82,4).

\section{Discusión}

Diversos estudios han demostrado que MINS es de importancia pronóstica en pacientes sometidos a cirugía no cardiaca[5],[6] MINS se ha asociado con un aumento significativo de la mortalidad a los 30 días[5],[6] y a más largo plazo[8]. Sin embargo, las guías 2014 de evaluación cardiovascular preoperatoria de American College of Cardiology/American Heart Association (ACC/AHA)[1], concluyen que la medición de rutina de los niveles de troponina postoperatoria en pacientes sin síntomas ni signos de isquemia miocárdica o infarto de miocardio no son útiles para guiar el manejo perioperatorio. Tampoco recomiendan la vigilancia postoperatoria de troponina en pacientes sin síntomas ni signos de isquemia miocárdica, con alto riesgo de infarto de miocardio (como pacientes sometidos a cirugía vascular)[1].

Estas conclusiones se basan en la ausencia de una estrategia clara de manejo o tratamiento a seguir con 
los pacientes que desarrollan MINS[1].

Foucrier y cols.[9], desarrollaron un estudio de casos y controles de pacientes quirúrgicos vasculares, en el cual concluyeron que la optimización de la terapia médica en pacientes con elevación de troponina postoperatoria se asoció con una mayor sobrevida a 12 meses. Las guías de 2017 de la sociedad cardiovascular canadiense sobre riesgo cardíaco perioperatorio, evaluación y tratamiento para pacientes de cirugía no cardíaca[10] recomiendan obtener mediciones diarias de troponina durante 48 a 72 horas después de cirugía no cardíaca, en pacientes con un riesgo basal superior al 5\% de muerte cardiovascular o infarto agudo al miocardio no fatal a 30 días después de una cirugía. Esto aplicaría a pacientes mayores de 65 años, entre 45 a 64 años con enfermedad cardiovascular significativa, a pacientes con un índice de riesgo cardíaco revisado (RCRI) de 1 y a los que tienen una concentración plasmática preoperatoria elevada de péptidos natriuréticos cerebral (BNP)[10]. Un análisis fármaco económico de Torborg y cols. [11], de la detección de troponina postoperatoria de rutina, sugiere que dicha vigilancia puede ser rentable[11].

\section{Conclusiones}

La injuria miocárdica posterior a cirugía no cardiaca se asocia con un mayor riesgo de mortalidad a 30 días -5 .

La vigilancia con troponina detectaría MINS en aproximadamente un $20 \%$ de los pacientes sometidos a cirugía vascular.

Un $12,5 \%$ de los pacientes que desarrollan MINS muere dentro de los 30 días de una cirugía vascular, pero aproximadamente un $75 \%$ de los casos de MINS no serán detectados sin la medición rutinaria de troponina post operatoria[7].

MINS es un marcador potencialmente útil de outcomes postoperatorios adversos y su detección podría brindar oportunidades para mejorar los resultados clínicos en los pacientes afectados.

\section{Referencias}

1. Fleisher LA, Fleischmann KE, Auerbach AD, Barnason SA, Beckman JA, Bozkurt B, et al.; American College of Cardiology; American Heart Association. 2014 ACC/AHA guideline on perioperative cardiovascular evaluation and management of patients undergoing noncardiac surgery: a report of the American College of Cardiology/ American Heart Association Task Force on practice guidelines. J Am Coll Cardiol. 2014 Dec;64(22):e77-137. https://doi. org/10.1016/j.jacc.2014.07.944 PMID:25091544

2. Bertges DJ, Goodney PP, Zhao Y, Schanzer A, Nolan BW, Likosky DS, et al. Vascular Study Group of New England. The Vascular Study Group of New England Cardiac Risk Index (VSG-CRI) predicts cardiac complications more accurately than the Revised Car- diac Risk Index in vascular surgery patients. J Vasc Surg. 2010 Sep;52(3):674-83. https://doi. org/10.1016/j.jvs.2010.03.031 PMID:20570467

3. Devereaux PJ, Chan MT, AlonsoCoello P, Walsh M, Berwanger O, Villar JC, et al. Vascular Events In Noncardiac Surgery Patients Cohort Evaluation (VISION) Study Investigators. Association between postoperative troponin levels and 30-day mortality among patients undergoing noncardiac surgery. JAMA. 2012 Jun;307(21):2295-304. https:// doi.org/10.1001/jama.2012.5502 PMID:22706835

4. Levy $M$, Heels-Ansdell $D$, Hiralal R, Bhandari M, Guyatt G, Yusuf $S$, et al. Prognostic value of troponin and creatine kinase muscle and brain isoenzyme measurement after noncardiac surgery: a systematic review and meta-analysis. Anesthesiology. 2011 Apr;114(4):796-
806. https://doi.org/10.1097/

ALN.0b013e31820ad503

PMID:21336095

5. van Waes JA, Nathoe HM, de Graaff JC, Kemperman H, de Borst GJ, Peelen LM, et al.; Cardiac Health After Surgery (CHASE) Investigators. Myocardial injury after noncardiac surgery and its association with shortterm mortality. Circulation. 2013 Jun;127(23):2264-71. https://doi. org/10.1161/CIRCULATIONAHA. 113.002128 PMID:23667270

6. Botto F, Alonso-Coello P, Chan MT, Villar JC, Xavier D, Srinathan S, et al.; Vascular events In noncardiac Surgery patlents cOhort evaluatioN (VISION) Writing Group, on behalf of The Vascular events In noncardiac Surgery patlents cOhort evaluatioN (VISION) Investigators; Appendix 1. The Vascular events In noncardiac Surgery patlents cOhort evaluatioN (VISION) Study Investigators Writing Group; 
Appendix 2. The Vascular events In noncardiac Surgery patlents cOhort evaluatioN Operations Committee; Vascular events In noncardiac Surgery patlents cOhort evaluatioN VISION Study Investigators. Myocardial injury after noncardiac surgery: a large, international, prospective cohort study establishing diagnostic criteria, characteristics, predictors, and 30-day outcomes. Anesthesiology. 2014 Mar;120(3):56478. https://doi.org/10.1097/ ALN.0000000000000113 PMID:24534856

7. Biccard BM, Scott DJ, Chan MT, Archbold A, Wang CY, Sigamani A, et al. Myocardial Injury After Noncardiac Surgery (MINS) in Vascular Surgical Patients: A Prospective Obser- vational Cohort Study. Ann Surg. 2018 Aug;268(2):35763. https://doi.org/10.1097/ SLA.0000000000002290 PMID:28486392

8. Kim M, Son M, Lee DH, Park K, Park TH. Troponin-l level after major non cardiac surgery and its association with long-term mortality. Int Heart J. 2016 May;57(3):278-84. https:// doi.org/10.1536/ihj.15-352 PMID:27150001

9. Foucrier $A$, Rodseth $R$, Aissaoui M, Ibanes C, Goarin JP, Landais $P$, et al. The long-term impact of early cardiovascular therapy intensification for postoperative troponin elevation after major vascular surgery. Anesth Analg. 2014 Nov;119(5):105363. https://doi.org/10.1213/
ANE.0000000000000302

PMID:24937347

10. Duceppe E, Parlow J, MacDonald $P$, Lyons $K$, McMullen $M$, Srinathan $S$, et al. Canadian Cardiovascular Society guidelines on perioperative cardiac risk assessment and management for patients who undergo noncardiac surgery. Can J Cardiol. 2017 Jan;33(1):17-32. https://doi. org/10.1016/j.cjca.2016.09.008 PMID:27865641

11. Torborg A, Ryan L, Kantor G, Biccard BM. The pharmacoeconomics of routine postoperative troponin surveillance to prevent and treat myocardial infarction after non-cardiac surgery. S Afr Med J. 2014 Jul;104(9):61923. https://doi.org/10.7196/ SAMJ.7654 PMID:25212403 